\section{Unmasking an oncometabolite}

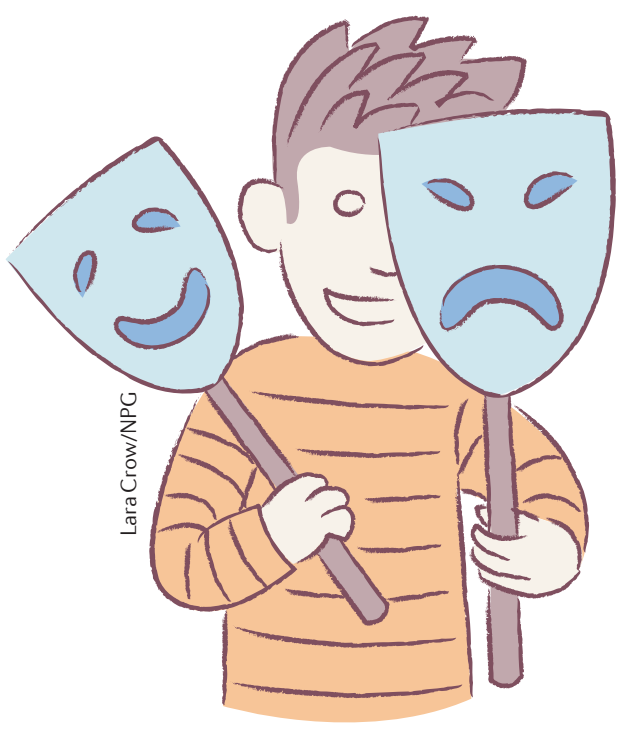

Isocitrate dehydrogenase 1 (IDH1) and IDH2 are mutated in a subset of gliomas and leukaemias. Mutation of these enzymes endows them with a new function: the generation of 2-hydroxyglutarate $(2 \mathrm{HG})$ instead of $\alpha$-ketoglutarate $(\alpha-K G)$. New data indicate that $2 \mathrm{HG}$ alters gene transcription through effects on DNA and histone methylation and hypoxia inducible factor $1 \alpha$ (HIF $1 \alpha)$ and HIF2a.

Gliomas with IDH mutations have a CpG island methylator phenotype (CIMP), and to determine whether these facts are linked, Timothy Chan and colleagues compared the DNA methylation patterns in isogenic astrocytes expressing either wild-type IDH1 or mutant IDH1. Expression of mutant IDH1 caused changes in the DNA methylome: mainly hypermethylation

2HG alters gene transcription through effects on DNA and histone methylation and hypoxia inducible factor $1 \alpha(\mathrm{HIF} 1 \alpha)$ and HIF $2 \alpha$.

IDH1 and gliomas with CIMP. In
addition, a gene expression signature

comprising 17 genes that are downregulated in cells expressing mutant IDH1 was able to classify an independent set of LGGs into CIMP positive or negative, indicating that IDH mutations and the CIMP are closely associated. Chromatin immunoprecipitation experiments indicated that the genes that are hypermethylated in cells expressing mutant IDH1 are associated with alterations in $\mathrm{H} 3 \mathrm{~K} 9$ and $\mathrm{H} 3 \mathrm{~K} 27$ histone methylation, indicating that alterations in histone marks might contribute to DNA hypermethylation. These authors also found that astrocytes expressing mutant IDH1 develop a stem-cell-like phenotype in concert with DNA hypermethylation, indicating that mutant IDH1 might prevent differentiation.

A suppression of differentiation is reported in the paper by Craig Thompson and colleagues. Gene expression and gene ontology analyses indicated that astrocyte and glial cell differentiation genes were highly differentially expressed in cells with IDH mutations compared to those without. $2 \mathrm{HG}$ can function as a competitive inhibitor of a-KG-dependent Jumonji-C histone demethylases (JHDMs). Expression of either IDH1 or IDH2 mutants in 293 T cells resulted in an increase in the levels of histone methylation, especially in the repressive histone marks H3K9me3 and H3K27me3. Moreover, the differentiation of mouse 3T3-L1 cells into adipocytes was inhibited by the expression of mutant IDH2 or the presence of a cell-permeable form of $2 \mathrm{HG}$. These authors also noted that the levels of JMJD2C, an H3K9-specific JHDM, were increased in differentiating 3T3-L1 cells. An in vitro histone demethylation assay confirmed that JMJD2C removed H3K9me3 and $\mathrm{H} 3 \mathrm{~K} 9 \mathrm{me} 2$ marks in the presence of $\alpha-K G$, and this reaction was competitively inhibited by 2 HG. Small interfering RNAs against JMJD2C also inhibited adipocyte differentiation, indicating that the removal of $\mathrm{H} 3 \mathrm{~K} 9$ methylation marks from histones is required for differentiation. Expression of mutant IDH1 in immortalized normal human astrocytes increased H3K9 methylation marks from passage 12 , and the expression of mutant IDH1 R132H in primary mouse neurosphere cultures prevented their differentiation.

In addition to the changes in DNA and histone methylation, William Kaelin and colleagues have found that unlike the $S$-enantiomer of $2 \mathrm{HG}$, which is inhibitory, the $R$-enantiomer, which is the form produced by mutant IDH1 and IDH2, can promote the activity of the HIFa prolyl 4-hydroxylases (EGLN1, EGLN2 and, to a lesser extent, EGLN3). (R)-2HG did not activate other $\alpha$-KG-dependent enzymes, including JMJD2D and the TET1 and TET2 methyl cytosine hydroxylases, all of which are inhibited by both $(S)-2 \mathrm{HG}$ and $(R)-2 \mathrm{HG}$. Further investigations revealed that (R)-2HG functions as a co-substrate for EGLN1 in the prolyl hydroxylation of HIF1a. Consistent with this, the stability of HIF $1 \alpha$ and HIF $2 \alpha$ was reduced in mid passage (9-15) human astrocytes expressing mutant IDH1, and a blunted HIF1a response to hypoxia was evident in cell lines derived from two oligodendrogliomas with IDH1 R132H mutations and in isogenic HCT116 colorectal cancer cell lines in which the $\mathrm{R} 132 \mathrm{H}$ mutant was expressed from the endogenous IDH1 locus. In addition, downregulation of HIF1a using short hairpin RNAs in immortalized human astrocytes increased their growth in soft agar, and a similar effect was seen when EGLN1 was overexpressed in these cells. Conversely, knockdown of EGLN1 in late passage cells expressing mutant IDH1 inhibited proliferation. Gene expression data from The Cancer Genome Atlas also showed that proneural tumours with IDH mutations had reduced expression levels of HIF-responsive genes. These authors conclude that the accumulation of $(R)-2$ HG promotes transformation through a mechanism that involves reduced HIF expression.

ORIGINAL RESEARCH PAPERS Turcan, S. et al. IDH1 mutation is sufficient to establish the glioma hypermethylator phenotype. Nature 15 Feb 2012 (doi:10.1038/nature10866) | Lu, C. et al. IDH mutation impairs histone demethylation and results in a block to cell differentiation. Nature 15 Feb 2012 (doi:10.1038/nature10860)| Koivunen, P. et al. Transformation by the $(R)$-enantiomer of 2-hydroxyglutarate linked to EGLN activation. Nature 15 Feb 2012 (doi:10.1038/nature10898) 